\title{
Relation between the rigorous and Helmert's definitions of orthometric heights
}

\author{
M. C. Santos (corresponding author) \\ Department of Geodesy and Geomatics Engineering, University of New Brunswick, P.O. \\ Box 4400, Fredericton, N.B., E3B 5A3, Canada; Phone: +1 506-453-4671; Fax: +1 506- \\ 453-4943; E-mail: msantos@unb.ca

\section{P. Vaníček} \\ Department of Geodesy and Geomatics Engineering, University of New Brunswick, P.O. \\ Box 4400, Fredericton, N.B., E3B 5A3, Canada; Phone: +1 506-453-5144; Fax: + 1 506- \\ 453-4943; E-mail: vanicek@unb.ca
}

\section{W. E. Featherstone}

Western Australian Centre for Geodesy, Curtin University of Technology, GPO Box U1987, Perth, WA 6845, Australia; Phone: +61 89266 2734; Fax: +61 89266 2703;

Email: W.Featherstone@curtin.edu.au

\section{R. Kingdon}

Department of Geodesy and Geomatics Engineering, University of New Brunswick, P.O. Box 4400, Fredericton, N.B., E3B 5A3, Canada; Phone: +1 506-458-7501; Fax: +1 506453-4943; Email: w4etp@unb.ca

\author{
A. Ellmann \\ Department of Geodesy and Geomatics Engineering, University of New Brunswick, P.O. \\ Box 4400, Fredericton, N.B., E3B 5A3, Canada; Phone: +1 506-458-7167; Fax: +1 506- \\ 453-4943; Email: ellmann@unb.ca

\section{B.-A. Martin} \\ Department of Geodesy and Geomatics Engineering, University of New Brunswick, P.O. \\ Box 4400, Fredericton, N.B., E3B 5A3, Canada \\ Currently at: Critchlow Associates Ltd., 61 Molesworth Street, Wellington, New Zealand
}

\section{Kuhn}

Western Australian Centre for Geodesy, Curtin University of Technology, GPO Box U1987, Perth, WA 6845, Australia; Phone: +61 89266 7603; Fax: +61 89266 2703;

Email: M.Kuhn@curtin.edu.au

\section{R. Tenzer}

School of Civil Engineering and Geosciences, University of Newcastle upon Tyne, Cassie Building, Newcastle upon Tyne, NE1 7RU, England; Phone: +44-191-2226399; Fax: +44-191-2228691; Email: robert.tenzer@ncl.ac.uk 


\begin{abstract}
Following our earlier definition of the rigorous orthometric height (Tenzer et al. 2005, J Geod, 79), we present the derivation and calculation of the differences between this and the Helmert orthometric height, which is embedded in the vertical datums used in numerous countries. By way of comparison, we also consider Mader and Niethammer's refinements to the Helmert orthometric height. For a profile across the Canadian Rocky Mountains (maximum height of $\sim 2,800 \mathrm{~m}$ ), the rigorous correction to Helmert's height reaches $\sim 13 \mathrm{~cm}$, whereas the Mader and Niethammer corrections only reach $\sim 3 \mathrm{~cm}$. The discrepancy is due mostly to the rigorous correction's consideration of the geoid-generated gravity disturbance. We also point out that several of the terms derived here are the same as those used in regional gravimetric geoid models, thus simplifying their implementation. This will enable those who currently use Helmert orthometric heights to upgrade them to a more rigorous height system based on the Earth's gravity field and one that is more compatible with the regional geoid model.
\end{abstract}

Keywords: Orthometric height, geoid, mean gravity, plumbline

\title{
1. Introduction
}

The orthometric height is defined as the metric length along the curved plumbline from the geoid to the Earth's surface. To calculate an orthometric height from spirit-levelling data and/or geopotential numbers requires that the mean value of the gravity along the plumbline between the Earth's surface and the geoid be known. This mean value is strictly defined in an integral sense (e.g., Heiskanen and Moritz 1967, p 166).

In the past, three main approximations have been applied in practice to evaluate this integral-mean value of gravity. The Helmert method, as described in Heiskanen and Moritz (1967, chap 4), applies the simplified Poincaré-Prey vertical gradient of gravity, which uses normal gravity and a Bouguer shell of constant topographic mass-density, to the observed gravity at Earth's surface in order to obtain an approximated mean value halfway down the plumbline. Niethammer (1932) and Mader (1954) refined Helmert's model by including the effect of local variations in the shape of the topography relative to the Bouguer shell. Mader, considering only the linear change of the gravimetric terrain correction with respect to depth, used the simple mean of the terrain effect at the geoid 
and at the Earth's surface, whereas Niethammer used the integral mean of terrain effects evaluated at discrete points along the plumbline. Dennis and Featherstone (2003) evaluated these three approximations, showing that the accuracy is ordered Niethammer, Mader then Helmert, which reflects the levels of approximation used.

In addition, the mean topographical mass-density $\rho_{0}=2670 \mathrm{kgm}^{-3}$, used in the Helmert, Niethammer and Mader approximations of the actual distribution of topographical mass-density, is not sufficiently accurate. (In his original manuscript, Helmert (1890) refers to a mass-density value of $2400 \mathrm{kgm}^{-3}$.) Attempts to refine the Helmert orthometric height in this regard have included varying topographical massdensity data (e.g., Sünkel 1986; Allister and Featherstone 2001) and borehole gravimetry (Strange 1982) to better approximate the integral-mean of gravity along the plumbline. To the best of our knowledge, no attempts have been made to include topographical mass-density data in the Mader and Niethammer heights (even though Niethammer (1930) already mentioned the necessity to use varying density information for a more rigorous treatment).

In this paper, we show that to arrive at a more rigorous orthometric height, one must take into account not only the effect of the shape of the topography and normal gravity, but also those additional effects coming from the masses contained within the geoid (herein termed the geoid-generated gravity) not accounted for by the Helmert approach and from the mass-density variations within the topography. This is necessary because the mean value of gravity along the plumbline between the geoid and the Earth's surface depends on all these quantities (cf. Tenzer et al. 2005). Mean gravity along the plumbline is thus evaluated as the sum of the integral-mean values of the geoid-generated gravity and the topography-generated gravitational attraction. For practical evaluation, the geoid-generated gravity is further divided into normal gravity and the geoid-generated gravity disturbance, i.e., the gravity disturbance in the so-called no-topography (NT) space (Vaníček at al. 2004). Likewise, the topography-generated gravity is divided among the spherical Bouguer shell, the terrain roughness residual to the Bouguer shell, and the topographical mass-density variations.

The aim of this paper is to provide the theoretical background and practical methods with which to convert Helmert orthometric heights (as described in, e.g., 
Heiskanen and Moritz (1967, chap 4)), which are used as the height system embedded in the vertical datum adopted in numerous countries, to the more rigorous orthometric heights presented in Tenzer et al. (2005). With this in mind, we have presented some preliminary derivations and results for various components of the correction in Vaníček et al. (2001) and in Tenzer and Vaníček (2004). Kingdon et al. (2005) present a numerical evaluation over part of Canada. This paper now presents the complete methodology.

\subsection{Notation and terminology}

In the sequel, the dummy argument $\Omega$ represents the geocentric spherical coordinates $\phi$ and $\lambda$ of a point $[\phi \in\langle-\pi / 2, \pi / 2\rangle, \lambda \in\langle 0,2 \pi\rangle]$ and $r$ denotes its geocentric radius. The radius of a point is a function of 3D location being represented by $r=r(\Omega)$. The symbols $r_{\mathrm{g}}(\Omega)$ and $r_{\mathrm{t}}(\Omega)$ represent the geocentric radii of the geoid and the Earth's surface, respectively, and will be abbreviated to $r_{\mathrm{g}}$ and $r_{\mathrm{t}}$ where there is no ambiguity. The orthometric height of a point is also a function of location, and is represented by $H^{\mathrm{O}}(\Omega)$.

The gravity at a point is a function of both the radius and the horizontal geocentric coordinates $\Omega$, being represented by $g(r(\Omega), \Omega)$ or in a simplified form used throughout the paper as $g(r, \Omega)$. The remaining gravity-related notation used throughout this paper is summarised in Table 1. Where relevant, overbars will be used to denote the integralmean quantities between the geoid and the Earth's surface.

\section{Table 1 near here}

\section{Recapitulation of the rigorous orthometric height}

The orthometric height $H^{O}(\Omega)$ of a point on the Earth's surface $\left(r_{t}, \Omega\right)$ is defined as the length of the curved plumbline between the geoid $r_{g}(\Omega)$ and the Earth's surface $r_{t}(\Omega) \cong r_{g}(\Omega)+H^{\mathrm{O}}(\Omega)$, and is given by (e.g., Heiskanen and Moritz 1967, Eq. 421):

$H^{O}(\Omega)=\frac{C\left(r_{t}, \Omega\right)}{\bar{g}(\Omega)}$ 
where $\bar{g}(\Omega)$ is the integral-mean integral value of gravity along the plumbline between the geoid and the Earth's surface:

$\bar{g}(\Omega)=\frac{1}{H^{\mathrm{O}}(\Omega)} \int_{r_{g}}^{r_{t}} g(r, \Omega) d r$

and $C\left(r_{t}, \Omega\right)$ is the geopotential number, which is the difference between the Earth's gravity potential $W_{0}[=$ constant $]$ at the geoid and $W\left(r_{t}, \Omega\right)$ at the Earth's surface:

$C(r, \Omega)=W-W(r, \Omega)$.

Concerning $\bar{g}(\Omega)$, since the actual value of gravity $g(r, \Omega)$ along the plumbline cannot be measured at all points, the integral-mean gravity $\bar{g}(\Omega)$ has to be computed from the observed surface gravity $g\left(r_{t}, \Omega\right)$, together with some realistic and physically meaningful model of $g(r, \Omega)$ along the plumbline. This computation can be achieved in practice by reducing the observed gravity according to some accepted model of the shape of the topography and the topographical mass-density distribution between the geoid and the Earth's surface.

\section{Decomposition of actual gravity}

In order to formulate the corrections to Helmert's orthometric height in a way that can be computed from the datasets currently available (i.e., terrestrial gravity observations, a digital elevation model and lateral density variations interpreted from geological maps or databases), we use the following decomposition of gravity. The primary pragmatic benefit of this approach is that these are the same data used to compute a geoid model, thus making the rigorous orthometric heights more compatible with the geoid model, provided of course the same corrections have been computed from the same data.

The gravity acceleration at a point $g(r, \Omega)$ can be decomposed into two terms; one comprising gravity generated by the masses inside geoid $g^{N T}(r, \Omega)$, i.e., in the NT-space (Vaníček et al. 2004), and another comprising the gravitation generated by topography $g^{T}(r, \Omega)$ :

$$
g(r, \Omega)=g^{N T}(r, \Omega)+g^{T}(r, \Omega) .
$$


Figure 1 schematically shows a cross-section of the Earth with the decomposition in Eq. (4), where the white internal area shows the contribution that comes from all masses within the geoid and the dark area shows the contribution due to the topographic masses.

\section{Figure 1 near here}

The geoid-generated gravity can be further decomposed into the contribution from normal gravity and the gravity disturbance caused by only the masses inside the geoid, the so-called NT gravity disturbance (cf. Vaníček et al. 2004). Likewise, the topographygenerated gravitation can be further decomposed into the Bouguer shell contribution and the terrain roughness term, residual to this shell. These two terms can also be adapted to include [lateral] topographical mass-density variations from the standard value of $\rho_{0}=2670 \mathrm{kgm}^{-3}$ (Section 5.5), this being the way in which topographic mass-density data is normally derived from geological maps.

The geoid-generated gravity is represented by the sum of normal gravity $\gamma(r, \Omega)$ and the geoid-generated gravity disturbance $\delta g^{N T}(r, \Omega)$ :

$g^{N T}(r, \Omega)=\gamma(r, \Omega)+\delta g^{N T}(r, \Omega)$.

The topography-generated gravitation is represented by the sum of that generated by Bouguer shell $g_{B}^{T}(r, \Omega)$, the topographical roughness residual to the Bouguer shell $g_{R}^{T}(r, \Omega)$, and the lateral variations in mass-density from the assumed average $\left(\rho_{0}=2670\right.$ $\left.\mathrm{kgm}^{-3}\right)$ within the topography $g \delta \rho(r, \Omega)$ :

$$
g^{T}(r, \Omega) \approx g_{B}^{T}(r, \Omega)+g_{R}^{T}(r, \Omega)+g^{\delta \rho}(r, \Omega) .
$$

Inserting Eqs. (5) and (6) into Eq. (4), gives a complete expression representing the total gravity as (cf. Tenzer et al. 2005):

$$
g(r, \Omega) \approx \gamma(r, \Omega)+\delta g^{N T}(r, \Omega)+g_{B}^{T}(r, \Omega)+g_{R}^{T}(r, \Omega)+g^{\delta \rho}(r, \Omega) .
$$

The approximation sign reflects the fact that two additional effects are omitted from Eq. (6) and (7): the gravitational effects of atmospheric masses and the radial variation of the topographic mass-density. The former is omitted because it is very small (cf. Tenzer et al. 2005, Appendix B); the latter is very difficult to quantify because there is not enough 
reliable information on the radial distribution of mass-density within the topography. As such, we shall only consider lateral topographic mass-density variations (cf. Martinec 1993). This is also consistent with the treatment of the geoid in the Stokes-Helmert scheme (e.g., Vaníček and Martinec 1994).

Finally, the integral-mean gravity along the plumbline $\bar{g}(\Omega)$, given by the integral-mean of $g(r, \Omega)$ according to Eq. (2) when applied to Eq. (7), is:

$\bar{g}(\Omega) \approx \bar{\gamma}(\Omega)+\overline{\delta g}^{N T}(\Omega)+\bar{g}_{B}^{T}(\Omega)+\bar{g}_{R}^{T}(\Omega)+\bar{g}^{\delta g}(\Omega)$.

\section{Helmert's and other approximations of the orthometric height}

By way of comparison, the expression for the approximated mean gravity along the plumbline used in the Helmert orthometric height (i.e., computed using the simplified Poincaré-Prey reduction), is (cf. Heiskanen and Moritz 1967, Eq. 4-25):

$\bar{g}^{H}(\Omega)=g\left(r_{t}, \Omega\right)-\frac{1}{2}\left(\frac{\partial \gamma}{\partial h}+4 \pi G \rho_{0}\right) H^{O}(\Omega)$,

where $\partial \gamma / \partial h$ is the linear vertical gradient of normal gravity, evaluated at the surface, $G$ is the Newtonian gravitational constant, and $\rho_{0}$ is the [assumed-constant] topographical mass-density.

It is worth mentioning that, in this paper, we follow the expression for Helmert's orthometric height (Eq. 9) as given in Heiskanen and Moritz (1967, chap 4). This is of most interest because this is the way in which most (if not all) geodesists have assumed Helmert's definition, and using a planar approximation of the terrain. In his original work, however, Helmert (1890) considered the gravitational effect of the complete topographic masses, delineating that the varying density within the topographic masses and the masses below the geoid should be considered in a rigorous treatment. While this is described in Helmert's (1890) text, his mathematical formulation is simpler, thus explaining why the simplification in Eq. (9) has been adopted in practice.

Using the numerical values of $\partial \gamma / \partial h=-0.3086 \mathrm{mGal} / \mathrm{m}$ and $2 \pi G \rho_{0}=+0.1119$ $\mathrm{mGal} / \mathrm{m}$ (the linear vertical gravity gradient from the Bouguer shell for $\rho_{0}=2670 \mathrm{~kg} / \mathrm{m}^{3}$ ) in Eq. (9) gives: 
$\bar{g}^{H}(\Omega)=g\left(r_{t}, \Omega\right)+0.0424 H^{O}(\Omega)$.

Therefore, Eqs. (9) and (10) effectively attempt to reduce surface gravity to a point halfway down the plumbline, using the Poincaré-Prey approximation of the vertical gravity gradient, to give an approximation of the integral-mean value along the plumbline between the geoid and Earth's surface. Note that this approximation embeds a constant topographic mass-density for the Bouguer shell and completely neglects the shape of the topography residual to the Bouguer shell. Making use of the general Eq. (7) at the Earth's surface (i.e., $r=r_{t}$ ), from Eq. (9) we obtain:

$$
\begin{aligned}
\bar{g}^{H}(\Omega) & =\gamma\left(r_{t}, \Omega\right)+\delta g^{N T}\left(r_{t}, \Omega\right)+g_{B}^{T}\left(r_{t}, \Omega\right)+g_{R}^{T}\left(r_{t}, \Omega\right) \\
& +g^{\delta \rho}\left(r_{t}, \Omega\right)-\frac{1}{2}\left(\frac{\partial \gamma}{\partial h}+4 \pi G \rho_{0}\right) H^{O}(\Omega)
\end{aligned}
$$

It is also worthwhile relating the rigorous orthometric height to the Niethammer (1932) and Mader (1954) orthometric heights. This is curiosity driven, since these height systems are not in wide practical use to the best of our knowledge. Both systems attempt to take the shape of the topography, residual to the Bouguer shell, into account when determining the integral-mean value of gravity along the plumbline. Both Mader and Niethammer orthometric heights include a term in the computation of mean gravity to include the mean terrain effect. Niethammer performs a discrete evaluation of the integral-mean terrain effect at a series of discrete points at even intervals along the plumbline, while Mader assumes the terrain effect to vary linearly between the geoid and the surface, and so uses a simple mean of the values of the effect evaluated for the Earth's surface and the geoid.

In our terminology, and using our approach to evaluate the terrain roughness term, Mader's (1954) approximated mean value of gravity along the plumbline method is:

$$
\bar{g}^{M}(\Omega)=\bar{g}^{H}(\Omega)+\frac{g_{R}^{T}\left(r_{g}, \Omega ; \rho_{0}\right)-g_{R}^{T}\left(r_{t}, \Omega ; \rho_{0}\right)}{2},
$$

and according to Niethammer's (1932) method, it is:

$$
\bar{g}^{N}(\Omega)=\bar{g}^{H}(\Omega)-g_{R}^{T}\left(r_{t}, \Omega ; \rho_{0}\right)+\frac{1}{H^{O}(\Omega)} \int_{r=R}^{R+H^{O}(\Omega)} g_{R}^{T}(r, \Omega) d r .
$$




\section{Corrections to the Helmert orthometric height}

To establish the relationships between the more rigorous mean gravity given by Eq. (8) and Helmert's approximate (Poincaré-Prey) formula given by Eq. (11), we subtract them, grouping like terms. The resulting difference is called the correction to Helmert's mean gravity $\mathcal{E}_{g}^{-}(\Omega)$ :

$$
\begin{aligned}
\mathcal{E}_{\bar{g}}(\Omega)= & \bar{g}(\Omega)-\bar{g}^{H}(\Omega) \\
\mathcal{E}_{\bar{g}}(\Omega)= & {\left[\bar{\gamma}(\Omega)-\gamma\left(r_{t}, \Omega\right)+\frac{1}{2} \frac{\partial \gamma}{\partial h} H^{O}(\Omega)\right] } \\
& +\left[\bar{g}_{B}^{T}(\Omega)-g_{B}^{T}\left(r_{t}, \Omega\right)+2 \pi G \rho_{0} H^{O}(\Omega)\right] \\
& +\left[\overline{\delta g}^{N T}(\Omega)-\delta g^{N T}\left(r_{t}, \Omega\right)\right]+\left[\bar{g}_{R}^{T}(\Omega)-g_{R}^{T}\left(r_{t}, \Omega\right)\right] \\
& +\left[\bar{g}^{\delta \rho}(\Omega)-g^{\delta \rho}\left(r_{t}, \Omega\right)\right]
\end{aligned}
$$

After being computed, $\mathcal{E}_{g}^{-}(\Omega)$ can be used to apply a correction to Helmert's orthometric height $\varepsilon_{H^{o}}(\Omega)$ using (cf. Heiskanen and Moritz 1967, p 169):

$$
\varepsilon_{H^{o}}(\Omega)=\frac{-H^{o}(\Omega)}{\bar{g}(\Omega)} \mathcal{E}_{\bar{g}}(\Omega),
$$

to an accuracy of $\ll 1 \mathrm{~mm}$ in $\mathcal{E}_{H^{o}}(\Omega)$. Since $\mathcal{E}_{\bar{g}}(\Omega)$ is small, the actual mean gravity in Eq. (15) can [ironically] be computed using Helmert's approximation (Eq. 10). This will make it considerably easier to numerically evaluate $\varepsilon_{H^{o}}(\Omega)$ in later sections:

$\varepsilon_{H^{o}}(\Omega)=\frac{-H^{o}(\Omega) \varepsilon_{\bar{g}}(\Omega)}{g\left(r_{t}, \Omega\right)+0.0424 H^{o}(\Omega)}$,

where $\varepsilon_{H^{o}}(\Omega)$ and $H^{o}(\Omega)$ are in metres, and $\varepsilon_{\bar{g}}(\Omega)$ and $g\left(r_{t}, \Omega\right)$ are in $\mathrm{mGal}$.

\subsection{Second-order correction for normal gravity}

For the terms involving normal gravity, we seek a simplification of

$$
A=\left[\bar{\gamma}(\Omega)-\gamma\left(r_{t}, \Omega\right)+\frac{1}{2} \frac{\partial \gamma}{\partial h} H^{o}(\Omega)\right]
$$


The integral-mean value of normal gravity along the plumbline $\bar{\gamma}(\Omega)$ is evaluated using a second-order Taylor expansion for the analytical downward continuation of normal gravity from the Earth's surface $\gamma\left(r_{t}, \Omega\right)$ to the geoid. Using a formulation in terms of geodetic coordinates, this is:

$\bar{\gamma}(\Omega) \approx \frac{1}{H^{O}(\Omega)} \int_{n=N(\Omega)}^{h(\Omega)=N(\Omega)+H^{o}(\Omega)}\left(\gamma(h(\Omega), \Omega)+\left.\frac{\partial \gamma}{\partial h}\right|_{h=h(\Omega)}[n-h(\Omega)]+\left.\frac{1}{2} \frac{\partial^{2} \gamma}{\partial h^{2}}\right|_{h=h(\Omega)}[n-h(\Omega)]^{2}\right) d n$

where $h(\Omega)$ is the geodetic [ellipsoidal] height of the point $\left(r_{t}, \Omega\right), N(\Omega)$ is the geoid height at $\Omega$, and $n$ is an element along the ellipsoidal plumbline (cf. Jekeli 2000). Performing the integration, applying the integration limits, and expressing normal gravity in terms of the geocentric radius of the Earth's surface at $\Omega$ gives:

$\bar{\gamma}(\Omega) \approx \gamma(h(\Omega), \Omega)-\left.\frac{1}{2} \frac{\partial \gamma}{\partial h}\right|_{h=h(\Omega)} H^{O}(\Omega)+\left.\frac{1}{6} \frac{\partial^{2} \gamma}{\partial h^{2}}\right|_{h=h(\Omega)}\left[H^{O}(\Omega)\right]^{2}$.

Inserting Eq. (19) in Eq. (17) yields:

$\left.A \approx \frac{1}{6} \frac{\partial^{2} \gamma}{\partial h^{2}}\right|_{h=h(\Omega)}\left[H^{O}(\Omega)\right]^{2}$.

Assuming the spherically approximated value of the second-order free-air gravity gradient (Heiskanen and Moritz 1967, Eq. 2-122), Eq. (20) reduces to:

$A=\gamma\left(\frac{H^{o}(\Omega)}{r_{t}(\Omega)}\right)^{2} \approx \gamma\left(\frac{H^{o}(\Omega)}{a}\right)^{2}$,

where $a$ is the major semi-axis of the reference ellipsoid. Taking $H^{O}(\Omega)=8.8 \mathrm{~km}$ (Mount Everest), $r_{t}(\Omega)=6371 \mathrm{~km}$ and $\gamma=9.81 \mathrm{~ms}^{-2}, A$ is about $-1.87 \mathrm{mGal}$. Using Eq. (16), this causes a maximum correction of about $1.5 \mathrm{~cm}$ to the Helmert orthometric height.

\subsection{Second-order correction for the Bouguer shell}

For the terms involving the spherical Bouguer shell of thickness $H^{o}(\Omega)$, we seek a simplification of the term 


$$
B=\left[\bar{g}_{B}^{T}(\Omega)-g_{B}^{T}\left(r_{t}, \Omega\right)+2 \pi G \rho_{0} H^{O}(\Omega)\right] .
$$

The gravitational attraction of the spherical Bouguer shell at the Earth's surface reads (Martinec 1993, Eq. 4.16):

$$
g_{B}^{T}\left(r_{t}, \Omega\right)=4 \pi G \rho_{0} H^{O}(\Omega) \frac{R^{2}}{\left(R+H^{O}(\Omega)\right)^{2}}\left(1+\frac{H^{O}(\Omega)}{R}+\frac{1}{3}\left(\frac{H^{o}(\Omega)}{R}\right)^{2}\right) .
$$

From Wichiencharoen (1982) (cited by Martinec 1998, Eq. 3.14), the gravitational potential inside the spherical Bouguer shell is:

$r_{g} \leq r \leq r_{t}: \quad V_{B}^{T}(r, \Omega)=2 \pi G \rho_{0}\left(\left[R+H^{O}(\Omega)\right]^{2}-\frac{2 R^{3}}{3 r}-\frac{r^{2}}{3}\right)$,

where $R$ is the radius of the base of the shell (in this case $R=r_{g}$ ) and $r$ is a dummy point inside the shell. Recognising that $g_{B}^{T}(r, \Omega)=-\frac{\partial V_{B}^{T}(r, \Omega)}{\partial r}$, the integral mean $\bar{g}_{B}^{T}(\Omega)$ in Eq. (22) along the radial between the geoid $r_{g}=R$ and approaching the Earth's surface $r_{t} \rightarrow R+H^{O}(\Omega)$ from within the Bouguer shell gives:

$\bar{g}_{B}^{T}(\Omega)=\frac{-1}{H^{O}(\Omega)} \int_{r=r_{g}}^{r_{t}-r_{g}+H(\Omega)} \frac{\partial V_{B}^{T}(r, \Omega)}{\partial r} d r \approx \frac{V_{B}^{T}\left(r_{g}\right)-V_{B}^{T}\left(r_{t}\right)}{H^{O}(\Omega)}$.

As for the normal gravity term, this is a more rigorous formulation for the spherical Bouguer shell, where $r$ and $H$ are along the same radial (i.e., $H^{O}(\Omega)=r_{t}(\Omega)-r_{g}(\Omega)$ ). As such, there is no need to worry about the deviation of the radial from the plumbline in this case (cf. Tenzer et al. 2005, Appendix A). Inserting the integration limits in Eq. (24), then inserting the results into Eq. (25), after some algebraic manipulation, gives:

$$
\bar{g}_{B}^{T}(\Omega)=2 \pi G \rho_{0} H^{o}(\Omega)\left(1-\frac{2 H^{o}(\Omega)}{3\left(R+H^{O}(\Omega)\right)}\right) .
$$

Here we acknowledge the typographical error in the first term of Tenzer et al. (2005, Eq. 21).

Inserting Eqs. (23) and (26) in Eq. (22) gives:

$$
B=\frac{4}{3} \pi G \rho_{0} \frac{H^{O}(\Omega)^{2}}{R+H^{O}(\Omega)}\left(2-\frac{H^{O}(\Omega)}{R+H^{O}(\Omega)}\right) .
$$


Using the earlier example of Mount Everest, a constant topographical mass-density of $\rho_{0}=2670 \mathrm{~kg} / \mathrm{m}^{3}$ and Eq. (16), the second-order Bouguer term (Eq. 27) affects the orthometric height by as much as $-1.6 \mathrm{~cm}$. Equation (14) now becomes:

$$
\begin{aligned}
\mathcal{E}_{g}(\Omega) & =\left[\overline{\delta g}^{N T}(\Omega)-\delta g^{N T}\left(r_{t}, \Omega\right)\right]+\left[\bar{g}_{R}^{T}(\Omega)-g_{R}^{T}\left(r_{t}, \Omega\right)\right] \\
& +\left[\bar{g}^{\delta g}(\Omega)-g^{\delta g}\left(r_{t}, \Omega\right)\right]-\gamma\left(\frac{H^{O}(\Omega)}{r_{t}(\Omega)}\right)^{2}+\frac{4 \pi G \rho_{0}}{3}\left(2-\frac{H^{O}(\Omega)}{R+H^{O}(\Omega)}\right),
\end{aligned}
$$

which represents the integral-mean value of gravity along the plumbline expressed in terms of corrections to Helmert's approximate mean value. These comprise: mean and surface effects on gravity coming respectively from masses inside the geoid, terrain roughness, laterally variable density distribution, second-order free-air effects, and second-order Bouguer shell effects. All these terms must be computed to apply a rigorous correction to Helmert's orthometric height.

\subsection{The geoid-generated gravity disturbance}

In this Subsection, we shall concentrate on the term

$C=\left[\overline{\delta g}^{N T}(\Omega)-\delta g^{N T}\left(r_{t}, \Omega\right)\right]$,

which deals with the corrections to the Helmert orthometric height coming from the geoid-generated gravity disturbance, comprising the mean value along the plumbline $\overline{\delta g}^{N T}(\Omega)$ and value on the Earth's surface $\delta g^{N T}\left(r_{t}, \Omega\right)$.

The integral-mean value of the geoid-generated gravity disturbance along the plumbline between the geoid and the Earth surface can be represented (in analogy to Eq. 2) by:

$$
\begin{aligned}
\overline{\delta g}^{N T}(\Omega) & =\frac{1}{H^{O}(\Omega)} \int_{r_{g}}^{r_{t}} \delta g^{N T}(r, \Omega) d r \\
& \cong \frac{1}{H^{O}(\Omega)} \int_{r=R}^{R+H^{O}} \delta g^{N T}(r, \Omega) d r
\end{aligned}
$$

where the geocentric radius of the geoid surface $r_{g}$ is approximated by $R$, the mean radius of the Earth, which should not be confused with the subscript $R$ in the 
topographical roughness term. Since the geoid-generated gravity disturbance $\delta g^{\mathrm{NT}}(r, \Omega)$ multiplied by $r$ is harmonic above the geoid (since the NT space contains no topographical masses above the geoid, and again neglecting the atmosphere), $\overline{\delta g}^{\mathrm{NT}}(\Omega)$ can be evaluated by averaging Poisson's equation for upward continuation (e.g., Kellogg 1929) in an integral sense. The Poisson equation reads:

$\delta g^{N T}(r, \Omega)=\frac{1}{4} \frac{R}{r} \iint_{\Omega^{\prime} \in \Omega_{0}} K\left[r, \psi\left(\Omega, \Omega^{\prime}\right), R\right] \delta g^{N T}\left(R, \Omega^{\prime}\right) d \Omega^{\prime}$,

where $\Omega_{0}$ is the solid angle, $\Omega^{\prime}$ is the dummy element and $\psi\left(\Omega, \Omega^{\prime}\right)$ represents the spherical distance or geocentric angle between the computation and integration points. The required gravity disturbance $\delta g^{N T}(R, \Omega)$ referred to the geoid is a part of the subintegral function. The spatial form of the Poisson integral kernel $K\left[r, \psi\left(\Omega, \Omega^{\prime}\right), R\right]$ is given by (e.g., Kellogg 1929):

$K\left[r, \psi\left(\Omega, \Omega^{\prime}\right), R\right]=R \frac{r^{2}-R^{2}}{\ell^{3}\left[r, \psi\left(\Omega, \Omega^{\prime}\right), R\right]}$,

where the Euclidean spatial distance is given by:

$\ell=\sqrt{r^{2}+r^{\prime 2}-2 r r^{\prime} \cos \psi\left(\Omega, \Omega^{\prime}\right)}$,

Inserting Eq. (31) into Eq. (30), the mean gravity disturbance $\overline{\delta g}{ }^{\mathrm{NT}}(\Omega)$ becomes (cf. Tenzer et al. 2005, Eq. 8):

$\overline{\delta g}^{N T}(\Omega) \cong \frac{1}{4 \pi} \frac{R}{H^{o}(\Omega)} \iint_{\Omega^{\prime} \in \Omega_{O}} \int_{r=R}^{R+H^{o}(\Omega)} \frac{1}{r} K\left[r, \psi\left(\Omega, \Omega^{\prime}\right), R\right] d r \delta g^{N T}\left(R, \Omega^{\prime}\right) d \Omega^{\prime}$.

Performing the radial integration of Poisson's integral kernel $K\left[r, \psi\left(\Omega, \Omega^{\prime}\right), R\right]$, multiplied by $r^{-1}$, the following expression can be found for the averaged Poisson's kernel (e.g., Vaníček at al. 2004, Tenzer et al. 2005):

$$
\begin{aligned}
& \left.\int_{r=R}^{R+H^{O}(\Omega)} \frac{1}{r} K\left[r, \psi\left(\Omega, \Omega^{\prime}\right), R\right)\right] d r=R \int_{r=R}^{R+H^{O}(\Omega)} \frac{1}{r} \frac{r^{2}-R^{2}}{\ell^{3}\left[r, \psi\left(\Omega, \Omega^{\prime}\right), R\right]} d r \\
& =\left|-\frac{2 R}{\ell\left[r, \psi\left(\Omega, \Omega^{\prime}\right), R\right]}+\ln \right| \frac{R-r \cos \psi\left(\Omega, \Omega^{\prime}\right)+\ell\left[r, \psi\left(\Omega, \Omega^{\prime}\right), R\right]}{r \sin \psi\left(\Omega, \Omega^{\prime}\right)}||_{r=R}^{R+H^{O}(\Omega)}
\end{aligned}
$$


for $\psi \neq 0$. Substituting Eq. (32) into Eq. (31), the mean gravity disturbance $\overline{\delta g}{ }^{\mathrm{NT}}(\Omega)$ along the plumbline takes the following form:

$$
\begin{aligned}
& \overline{\delta g}^{N T}(\Omega)=\frac{1}{4 \pi} \frac{R}{H^{O}(\Omega)} \iint_{\Omega^{\prime} \in \Omega_{0}}\left[\frac{2 R}{\ell\left[R, \psi\left(\Omega, \Omega^{\prime}\right), R\right]}-\frac{2 R}{\ell\left[r_{t}(\Omega), \psi\left(\Omega, \Omega^{\prime}\right), R\right]}\right. \\
& \left.+\ln \left|\frac{R}{r_{t}(\Omega)}\left(\frac{R-r_{t}(\Omega) \cos \psi\left(\Omega, \Omega^{\prime}\right)+\ell\left[r_{t}(\Omega), \psi\left(\Omega, \Omega^{\prime}\right), R\right]}{R\left(1-\cos \psi\left(\Omega, \Omega^{\prime}\right)\right)+\ell\left[R, \psi\left(\Omega, \Omega^{\prime}\right), R\right]}\right)\right|\right] \delta g^{N T}\left(R, \Omega^{\prime}\right) d \Omega^{\prime} .
\end{aligned}
$$

Equation (36) can be simplified as:

$$
\overline{\delta g}^{N T}(\Omega)=\frac{1}{4 \pi} \frac{R}{H^{O}(\Omega)} \iint_{\Omega^{\prime} \in \Omega_{O}} \bar{K}\left[R+H^{o}(\Omega), \psi\left(\Omega, \Omega^{\prime}\right), R\right] \delta g^{N T}\left(R, \Omega^{\prime}\right) d \Omega^{\prime}
$$

where $\bar{K}$ stands for the intermediary integration kernel. It can be shown, in the first approximation, that this kernel equals:

$\bar{K}\left[R+H(\Omega), \psi\left(\Omega, \Omega^{\prime}\right), R\right]=2 R\left(\frac{1}{\ell}-\frac{1}{\ell^{*}}\right)+\ln \frac{\ell^{*}-H}{\ell}$,

where $\ell$ stands for $\ell(R, \psi, R)$ and $\ell^{*}$ stands for $\ell\left(R+H^{O}(\Omega), \psi, R\right)$. The derivation is given in Appendix A.

Equation (37) is somewhat cumbersome because it requires that the NT gravity disturbance be known on the geoid, which it is not. Therefore, to implement it in practice first requires the downward continuation of $\delta g^{N T}\left(r_{t}, \Omega\right)$ to $\delta g^{N T}\left(r_{g}, \Omega\right)$. In Eq. (36), the geoid-generated gravity disturbance $\delta g^{\mathrm{NT}}\left(r_{g}, \Omega\right)$ is obtained from the geoidgenerated gravity anomaly $\Delta g^{\mathrm{NT}}\left(r_{g}, \Omega\right)$ referred to the geoid in the NT-space by (cf. Heiskanen and Moritz, 1967, Eq. 2-151e; Vaníček et al. 2004):

$\delta g^{N T}(R, \Omega)=\Delta g^{N T}(R, \Omega)+\frac{2}{R} T^{N T}(R, \Omega)$

where $T^{N T}(R, \Omega)$ represents the geoid-generated disturbing potential in the NT space:

$T^{N T}(R, \Omega)=T(R, \Omega)-V^{T}(R, \Omega)-V^{A}(R, \Omega)$ 
The disturbing potential $T(R, \Omega)$ can be taken from a regional geoid model, computed according to Bruns (1878) formula for the geoid height $T=N(\Omega) \gamma_{0}$, thus making the geoid and the corresponding orthometric height system more compatible.

The second term on the right-hand side of $\mathrm{Eq}(40)$ is the gravitational potential $V^{T}\left(r_{g}, \Omega\right)$ of the topographical masses, and $V^{A}\left(r_{g}, \Omega\right)$ is the potential of all atmospheric masses. The term $V^{T}\left(r_{g}, \Omega\right)$ is obtained through the Newtonian integral:

$$
V^{T}\left(r_{g}, \Omega\right)=G \iint_{\Omega^{\prime} \in \Omega_{0}} \int_{r^{\prime}=R}^{R+H^{0}\left(\Omega^{\prime}\right)} \rho\left(r^{\prime}, \Omega^{\prime}\right) \ell^{-1}\left[R, \psi\left(\Omega, \Omega^{\prime}\right), r^{\prime}\right] r^{\prime 2} d r^{\prime} d \Omega^{\prime},
$$

where $\rho\left(r^{\prime}, \Omega^{\prime}\right)$ represents the actual mass-density of the topographical masses, usually computed from a density distribution model. The effect due to lateral density variation is dealt with in subsection 5.5.

Finally, to complement Eq. (29), the gravity disturbance at the Earth's surface is required. This term can be evaluated directly from:

$$
\delta g^{N T}\left(r_{t}, \Omega\right)=\Delta g^{N T}\left(r_{t}, \Omega\right)+\frac{2}{r_{t}} T\left(r_{t}, \Omega\right)-\frac{2}{r_{t}} V^{T}\left(r_{t}, \Omega\right)-\frac{2}{r_{t}} V^{A}\left(r_{t}, \Omega\right) .
$$

Equation (29) can then be evaluated using Eqs. (37) and (42).

\subsection{The terrain-roughness-generated gravity}

In this Subsection, we shall concentrate on the term

$$
D=\left[\bar{g}_{R}^{T}(\Omega)-g_{R}^{T}\left(r_{t}, \Omega\right)\right]
$$

which gives the correction to the Helmert orthometric height from the terrain roughness residual to the Bouguer shell, assuming for the moment a constant topographical massdensity. (Lateral density variations will be considered in Subsection 5.5). The gravitational field of the topographical roughness term is not harmonic inside the topography. As such, it has to be calculated from an adopted model of the shape of the topography (i.e., a digital elevation model, DEM), coupled with a constant mass-density assumption. 
We begin with the gravitational potential $V^{T}(r, \Omega)$ of topographical masses expressed in Eq. (41). Using a spherical approximation of the geoid, this reads (cf. Novák and Grafarend 2005):

$V^{T}(r, \Omega) \approx G \iint_{\Omega^{\prime}}^{r^{\prime}=R+H^{o}\left(\Omega^{\prime}\right)} \int_{r^{\prime}=R} \rho\left(r^{\prime}, \Omega^{\prime}\right) \ell^{-1}\left(r, \Omega, r^{\prime}, \Omega^{\prime}\right) r^{\prime 2} d r^{\prime} d \Omega^{\prime}$.

The negative radial derivative of topographical gravitational attraction $g^{T}(r, \Omega)$ is given by:

$$
g^{T}(r, \Omega) \approx-G \iint_{\Omega^{\prime}} \frac{\partial}{\partial r}\left(\int_{r^{\prime}=R}^{r^{\prime}=R+H^{o}\left(\Omega^{\prime}\right)} \rho\left(r^{\prime}, \Omega^{\prime}\right) \ell^{-1}\left(r, \Omega, r^{\prime}, \Omega^{\prime}\right) r^{\prime 2} d r^{\prime}\right) d \Omega^{\prime} .
$$

From Eq. (43), we are looking for the mean value $\bar{g}^{T}(r, \Omega)$ between the Earth's surface and the geoid, which is given, by definition, as (Tenzer et al. 2005, Eqs. 16-18):

$$
\begin{aligned}
\bar{g}^{T}(r, \Omega) & =\frac{1}{H^{O}(\Omega)} \int_{r=R}^{r=R+H(\Omega)} g^{T}(r, \Omega) d r \\
& =\frac{-1}{H^{O}(\Omega)} \int_{r=R}^{r=R+H(\Omega)} \frac{\partial}{\partial r} V^{T}(r, \Omega) d r \\
& =\frac{-1}{H^{O}(\Omega)}\left\{V^{T}\left[R+H^{O}(\Omega), \Omega\right]-V^{T}[R, \Omega]\right\}
\end{aligned}
$$

Substituting for the two values of potential $V^{T}$ from Eq. (40), we get:

$$
\bar{g}^{T}(\Omega) \approx \frac{G}{H^{O}(\Omega)} \iint_{\Omega^{\prime}}^{R+H^{O}\left(\Omega^{\prime}\right)} \int_{r^{\prime}=R} \rho\left(r^{\prime}, \Omega^{\prime}\right)\left[\ell^{-1}\left[R, \Omega, r^{\prime}, \Omega \Omega^{\prime}\right]-\ell^{-1}\left[R+H^{O}(\Omega), \Omega, r^{\prime}, \Omega^{\prime}\right]\right] r^{\prime 2} d r^{\prime} d \Omega^{\prime} .
$$

Let us now express the radial integral in Eq. (47) as a sum of two integrals:

$$
\int_{R}^{R+H^{o}\left(\Omega^{\prime}\right)} F\left(r^{\prime}\right) d r^{\prime}=\int_{R}^{R+H^{o}(\Omega)} F\left(r^{\prime}\right) d r^{\prime}+\int_{R+H^{o}(\Omega)}^{R+H^{o}\left(\Omega^{\prime}\right)} F\left(r^{\prime}\right) d r^{\prime}
$$

The first integral on the right-hand side of Eq. (48) describes the contribution of the Bouguer shell of constant thickness $H^{\mathrm{O}}(\Omega)$ (e.g., Vaníček et al. 2001), which was dealt with in Subsection 5.2. The second integral gives the contribution due to the topographical roughness or terrain residual to the Bouguer shell. The density can also be written as a sum of two terms, one containing a contribution due to the mean density $\rho_{0}$ and the other containing the residual density $\delta \rho(r, \Omega)$ contribution: 
$\rho(r, \Omega)=\rho_{0}+\delta \rho(r, \Omega)$.

The roughness term is represented by the second term in Eq. (21) of Tenzer et al (2005):

$\bar{g}_{R}^{T}(\Omega)=\frac{G \rho_{0}}{H^{o}(\Omega)} \iint_{\Omega^{\prime} \in \Omega_{0}} \int_{r^{\prime}=R+H^{o}(\Omega)}^{R+H^{o}\left(\Omega^{\prime}\right)}\left\{\ell^{-1}\left[R, \Omega, r^{\prime}, \Omega^{\prime}\right]-\ell^{-1}\left[R+H^{o}(\Omega), \Omega, r^{\prime}, \Omega^{\prime}\right]\right\} r^{\prime 2} d r^{\prime} d \Omega^{\prime}$,

This term is nothing else but the change in the roughness part of the topography (also called "terrain" in some literature) of constant density of $\rho_{0}$, from the geoid to the surface of the Earth, divided by the orthometric height of the point of interest:

$\bar{g}_{R}^{T}\left(\Omega, \rho_{0}\right) \approx \frac{1}{H^{o}(\Omega)}\left[V_{R}^{T}(R, \Omega)-V_{R}^{T}\left(R+H^{O}(\Omega), \Omega\right)\right]$.

These two roughness parts of topographical potential can be evaluated through numerical quadrature of the Newton integral (Eq. 44). Equation (51) provides the mean gravity generated by the topographical roughness, expressed in terms of gravitational potential. As pointed out in Subsection 5.3, it comprises a contribution from the average topographical mass-density, plus a smaller correction due to mass-density variations.

The other term in Eq. (43), the topographical roughness term at the Earth's surface, is given by the second term in Martinec (1998):

$$
g_{R}^{T}\left(r_{t}, \Omega\right) \approx-\left.G \rho_{0} \iint_{\Omega^{\prime} \in \Omega_{0}} \int_{r^{\prime}=R+H^{o}(\Omega)}^{r^{\prime}=R+H^{o}\left(\Omega^{\prime}\right)} \frac{\partial \ell^{-1}\left(r, \Omega ; r^{\prime}, \Omega^{\prime}\right)}{\partial r}\right|_{r=r_{t}} r^{\prime 2} d r^{\prime} d \Omega^{\prime}
$$

which can also be evaluated by quadrature methods.

\subsection{The lateral variation of topographical mass-density}

In this Subsection, we consider the term:

$E=\left[\bar{g}^{\delta \rho}(\Omega)-g^{\delta \rho}\left(r_{t}, \Omega\right)\right]$.

In most gravimetric geoid computations, the topographical mass-density is generally modelled by an average value of $\rho_{0}=2670 \mathrm{kgm}^{-3}$. Martinec (1998) posed the question on how much a variation in topographical mass-density affects geoid height computation. To answer this question in the context of the orthometric height, we assume only lateral variations of density, leaving the radial variation still to be tackled. The developments below follow from those of Subsection 5.4. 
The contribution of lateral variation of density to the correction to Helmert's orthometric height is represented by third term in Eq. (21) from Tenzer et al (2005):

$\bar{g} \delta \rho(\Omega)=\frac{G}{H^{o}(\Omega)} \iint_{\Omega^{\prime} \in \Omega_{0}} \int_{r^{\prime}=R}^{r^{\prime}=R+H\left(\Omega^{\prime}\right)} \delta \rho\left(r^{\prime}, \Omega^{\prime}\right)\left\{\ell^{-1}\left[R, \Omega, r^{\prime}, \Omega^{\prime}\right]-\ell^{-1}\left[R+H(\Omega), \Omega, r^{\prime}, \Omega^{\prime}\right]\right\} r^{\prime 2} d r^{\prime} d \Omega^{\prime}$

The surface gravity generated by lateral variation of density is given by:

$g \delta \rho\left(r_{t}, \Omega\right)=-\left.G \iint_{\Omega \in \Omega_{0}} \delta \rho(\Omega) \int_{r=R}^{r=R+H\left(\Omega^{\prime}\right)} \frac{\partial \ell^{-1}\left[R, \Omega ; r^{\prime}, \Omega^{\prime}\right]}{\partial r}\right|_{r=r_{t}(\Omega)} r^{\prime 2} d r^{\prime} d \Omega^{\prime}$,

which follows from a more complete expression provided by Martinec (1998) that takes into account the radial variation in density $r^{\prime}$ :

$$
\left.g \delta \rho_{(}, \Omega\right)=-\left.G \iint_{\Omega^{\prime} \in \Omega_{0}} \int_{r^{\prime}=R}^{r^{\prime}=R+H\left(\Omega^{\prime}\right)} \delta \rho\left(r^{\prime}, \Omega^{\prime}\right) \frac{\partial \ell^{-1}\left[R, \Omega ; r^{\prime}, \Omega^{\prime}\right]}{\partial r}\right|_{r=r_{t}(\Omega)} r^{\prime 2} d r^{\prime} d \Omega^{\prime},
$$

Equations (54) and (55) provide the terms required in Eq. (53)

The correction to Helmert's orthometric height due to the laterally varying topographical mass-density is also given by the following approximate expression (Vaníček et al. 1995) if one considers only the radial gradient of the gravitational attraction generated by the spherical Bouguer shell of the anomalous topographical density $\delta \rho(\Omega)$ :

$\varepsilon_{H^{o}}^{\delta \rho}(\Omega) \approx 2 \pi G\left[H^{o}(\Omega)\right]^{2} \frac{\delta \rho(\Omega)}{g(\Omega)}$.

\subsection{Summary}

The correction to the Helmert orthometric height to give the rigorous orthometric height defined by Tenzer et al. (2005) $\varepsilon_{H^{O}}(\Omega)$ is given by Eq. (15). It follows directly from the evaluation of the correction to Helmert's mean gravity $\mathcal{E}_{g}(\Omega)$, written below in a simplified manner as:

$$
\mathcal{E}_{g}(\Omega)=A+B+C+D+E
$$

The terms $A$ and $B$ can be computed from Eq. (28) as: 
$A+B=-\gamma\left(\frac{H^{O}(\Omega)}{r_{t}(\Omega)}\right)^{2}+\frac{4 \pi G \rho_{0}}{3}\left(2-\frac{H^{O}}{R+H^{O}}\right)$

The terms $C, D$ and $E$ can be computed from Eqs. (37) and (42), (51) and (42) and (54) and (55), respectively. Note that several of these terms would have already been computed for a regional gravimetric geoid model based on the Stokes-Helmert approach (Vaníček and Martinec 1994). This simplifies the task, where the gridded quantities can be interpolated to the points of interest and applied as part of the corrections to the Helmert orthometric height. It also makes the rigorous orthometric heights more compatible with the regional geoid model. Finally, the total correction to the Helmert orthometric height $\varepsilon_{H^{O}}(\Omega)$ is:

$\varepsilon_{H} O(\Omega)=\frac{-H^{O}(\Omega)}{\bar{g}(\Omega)}(A+B+C+D+E)$

\section{Numerical Tests}

Using Canadian gravity, terrain and lateral topographic density data, we have computed rigorous corrections to Helmert's orthometric heights along a profile across the Canadian Rocky Mountains. This profile spans the longitudes from $235^{\circ} \mathrm{E}$ to $239^{\circ} \mathrm{E}$ along the $50^{\circ} \mathrm{N}$ parallel. Figure 2 shows each one of the terms in Eq. (60) (i.e., second-order freeair, second-order Bouguer shell, NT gravity disturbance, topographical roughness, and lateral density variations) computed separately to show their relative contributions to the correction. These terms are superimposed on the topographic height variations (shown with the thicker line in Fig. 2) scaled down by $100 \mathrm{~m}$, to show that there is not always a one-to-one correspondence of the correction terms with height. All integral terms were computed over a spherical cap radius of 3 degrees, beyond which the far-zone contributions become negligible $(<1 \mathrm{~mm})$ for this test area.

\section{Figure 2 near here}

Inspecting Fig. 2, we see that the correction term from the geoid-generated gravity disturbance gives the largest correction values, and is generally positively correlated with 
topography, though not perfectly. The correction due to terrain-roughness-generated gravity is the second most important contribution. However, it works against the former correction, and there is a less strong, negative correlation with topography. The third largest term in magnitude is the correction due to lateral variation of topographical massdensity, varying around zero and with maximum magnitude not greater than $5 \mathrm{~cm}$. The final two terms, due to second-order correction for normal gravity and second-order correction for the Bouguer shell, are both very small, not showing up in Fig. 2. Table 2 summarizes the statistics of these five correction terms.

\section{Table 2 near here}

Figure 3 shows a comparison among the corrections to Helmert orthometric heights using the method described in this paper (termed rigorous), and the Mader and Niethammer approaches, for the same profile as in Fig. 2. The Mader and Niethammer corrections were computed from Eqs. (12) and (13) using the same topographical corrections used to evaluate the rigorous corrections. From Fig. 3, the Mader and Niethammer corrections are very similar to one another, whereas the rigorous correction is larger, which is attributed to the two additional terms not accounted for in Mader nor Niethammer's approaches: geoid-generated gravity disturbance and lateral variation of topographical mass-density. The larger contribution comes mostly from the geoidgenerated gravity disturbance (cf. Fig. 2). Table 3 summarizes the statistics of the corrections along this profile.

Figure 3 and Table 3 near here

\section{Summary and Conclusions}

We have derived expressions to transform Helmert's orthometric height into a more rigorous one (cf. Tenzer et al. 2005), taking into account effects coming from the secondorder correction for normal gravity, second-order correction for the Bouguer shell, the geoid-generated gravity disturbance, the terrain-roughness-generated gravity and the lateral variation of topographical mass-density. These individual corrections have been 
evaluated numerically along a profile across the Canadian Rocky Mountains, and plotted against the topographical height variation.

This comparison shows that the geoid-generated gravity disturbance, the terrainroughness-generated gravity and the lateral variation of topographical mass-density are, respectively, the most important contributors towards obtaining a more rigorous orthometric height. It also shows that the geoid-generated gravity disturbance and the terrain-roughness-generated gravity work approximately against each other, though not completely as each is not perfectly correlated with the topography. The second-order correction for normal gravity and the second-order correction for the Bouguer shell are negligibly small for this test, but become larger for very high elevations.

Comparisons with other refinements of Helmert orthometric heights, namely Mader and Niethammer's, have also been performed. The Mader and Niethammer orthometric heights are very similar to one another, but the respective corrections are smaller than the rigorous corrections. They differ from the rigorous approach due to inclusion of the terms pertaining to the geoid-generated gravity anomaly and lateral variation of topographical mass-density.

Finally, it is important to point out that several of the correction terms used here are the same as would have been computed for a regional gravimetric geoid model based on the Stokes-Helmert approach. As such, they are relatively easy to apply to existing Helmert orthometric heights. Moreover, this makes the resulting heights more compatible with the gravimetric geoid model.

Acknowledgements: The Canadian investigators are supported by the "GEOIDE Network of Centres of Excellence". The Australian investigators are supported by Australian Research Council grant DP0211827. The topographical data were kindly supplied by Geodetic Survey Division of Natural Resources Canada.

\section{References}

Allister NA, Featherstone WE (2001) Estimation of Helmert orthometric heights using digital barcode levelling, observed gravity and topographic mass-density data over part of Darling Scarp, Western Australia, Geom Res Aust, 75:25-52 
Bruns H (1878) Die Figur der Erde, Publ. Preuss. Geod. Inst., Berlin

Dennis ML, Featherstone WE (2003) Evaluation of orthometric and related height systems using a simulated mountain gravity field, in: Tziavos IN (ed) Gravity and Geoid 2002, Dept of Surv and Geodesy, Aristotle Univ of Thessaloniki, pp. 389-394

Heiskanen WA, Moritz H (1967) Physical Geodesy, Freeman, San Francisco

Helmert FR (1890) Die Schwerkraft im Hochgebirge, Insbesondere in den Tyroler Alpen, Veröff. Königl. Preuss. Geod. Inst., No. 1, Berlin

Jekeli C (2000) Heights, the geopotential, and vertical datums, Rep 459, Dept Geod Sci, Ohio State Univ, Columbus

Kellogg OD (1929) Foundations of Potential Theory, J Springer, Berlin

Kingdon R, Vaníček P, Santos M, Ellmann A, Tenzer R (2005) Toward an improved orthometric height system for Canada, Geomatica (in press).

Mader K (1954) Die orthometrische Schwerekorrektion des Präzisions-Nivellements in den Hohen Tauern, Österreichische Zeitschrift für Vermessungswesen, Sonderheft 15 , Vienna

Martinec Z (1993) Effect of lateral density variations of topographical masses in view of improving geoid model accuracy over Canada, Final report of contract DSS No. 23244-2-4356, Geodetic Survey of Canada, Ottawa

Martinec Z (1998) Boundary value problems for gravimetric determination of a precise geoid. Lecture notes in Earth Sciences, 73, Springer, Berlin Heidelberg New York

Niethammer T (1932) Nivellement und Schwere als Mittel zur Berechnung wahrer Meereshöhen, Schweizerische Geodätische Kommission, Berne

Novák P, Grafarend EW (2005) Ellipsoidal representation of the topographical potential and its vertical gradient, J Geod 78(1-3):691-706, doi 10.1007/s00190-005-0435-4

Strange WE (1982) An evaluation of orthometric height accuracy using borehole gravimetry, Bull Géod, 56:300-311

Sünkel H (1986) Digital height and density model and its use for the orthometric height and gravity field determination for Austria, Proc Int Symp on the Definition of the Geoid, Florence, May, pp 599-604 
Tenzer R, Vaníček P, Santos M, Featherstone WE, Kuhn M (2005) Rigorous determination of the orthometric height, J Geod 79(1-3):82-92, doi: 10.1007/s00190005-0445-2

Vaníček P. Martinec Z (1994) Stokes-Helmert scheme for the evaluation of a precise geoid, manuscr geod 19(3):119-128

Vaníček P, Janák J, Huang JL (2001) Mean vertical gradient of gravity, in Sideris MG (ed.) Gravity, Geoid and Geodynamics 2000, Springer, Berlin Heidelberg New York, pp. $259-262$

Vaníček P, Novák P, Martinec Z (2001) Geoid, topography, and the Bouguer plate or shell, J Geod 75(4):210-215, doi: 10.1007/s001900100165

Vaníček P, Kleusberg A, Martinec Z, Sun W, Ong P, Najafi M, Vajda P, Harrie L, Tomášek P, ter Horst B (1995) Compilation of a precise regional geoid. Final report on research done for the Geodetic Survey Division, Fredericton

Vaníček P, Tenzer R, Sjöberg LE, Martinec Z, Featherstone WE (2004) New views of the spherical Bouguer gravity anomaly. Geophys J Int 159(2):460-472, doi: 10.1111/j.1365-246X.2004.02435.x

Wichiencharoen C (1982) The indirect effects on the computation of geoid undulations, Rep 336, Dept Geod Sci, Ohio State Univ, Columbus 


\section{Appendix A: Derivation of Eq. (38)}

We wish to simplify the expression for the averaging Poisson's kernel (Eq. 35), which reads:

$$
\begin{aligned}
& \bar{K}\left(R+H^{o}(\Omega), \psi, R\right)=-\frac{2 R}{\ell\left(R+H^{o}(\Omega), \psi, R\right)}+\frac{2 R}{\ell(R, \psi, R)}+ \\
& +\ln \left|\frac{R-\left(R+H^{o}(\Omega)\right) \cos \psi+\ell\left(R+H^{o}(\Omega), \psi, R\right)}{\left(R+H^{o}(\Omega)\right) \sin \psi}\right|-\ln \left|\frac{R-R \cos \psi+\ell(R, \psi, R)}{R \sin \psi}\right|
\end{aligned}
$$

For integration within a very small radius $\psi_{0}$ of, say 3 arc-degrees, we can assume:

$$
\ell, \ell^{*}, H^{O}(\Omega)<R,
$$

where we have denoted $\ell(R, \psi, R)$ by $\ell$ and $\ell\left(R+H^{O},(\Omega), \psi, R\right)$ by $\ell^{*}$. This is permitted because of the rapid decay of the Poisson kernel with spherical distance, as supported by our empirical evidence (cf. Section 6). Now, we can rewrite Eq. (A1) as:

$$
\bar{K}\left(R+H^{o}(\Omega), \psi, R\right)=2 R\left(\frac{1}{\ell}-\frac{1}{\ell^{*}}\right)+\ln \left|\frac{1-\left(1+H^{o}(\Omega) / R\right) \cos \psi+\ell^{*} / R}{\left(1+H^{o}(\Omega) / R\right)(1-\cos \psi+\ell / R)}\right| .
$$

The last term in Eq. (A3) should be:

$$
\ln \left|\frac{1-\left(1+H^{O}(\Omega) / R\right) \cos \psi+\ell^{*} / R}{\left(1+\frac{H^{O}(\Omega)}{R+H^{O}(\Omega)}\right)(1-\cos \psi+\ell / R)}\right| .
$$

However, due to the precision required, the approximation in Eq. (A3) is enough since:

$$
\left(1+\frac{H^{o}(\Omega)}{R+H^{o}(\Omega)}\right) \approx\left(1+\frac{H^{o}(\Omega)}{R}\right)
$$

Realizing that:

$$
\ell=2 R \sin \frac{\psi}{2},
$$

we can express $\cos \psi$ in Eq.(A4) as:

$$
\cos \psi=1-2 \sin ^{2} \frac{\psi}{2}=1-\frac{1}{2}\left(\frac{\ell}{R}\right)^{2} \text {. }
$$


Substituting this result into Eq.(A3), gives:

$$
\bar{K}\left(R+H^{O}(\Omega), \psi, R\right)=2 R\left(\frac{1}{\ell}-\frac{1}{\ell *}\right)+\ln \left|\frac{1-\left(1+H^{O}(\Omega) / R\right)\left[1-\frac{1}{2}\left(\frac{\ell}{R}\right)^{2}\right]+\ell * / R}{\left(1+H^{O}(\Omega) / R\right)\left\{1-\left[1-\frac{1}{2}\left(\frac{\ell}{R}\right)^{2}\right]+\ell / R\right\}}\right|
$$

After a few algebraic operations, we get:

$$
\begin{aligned}
\bar{K}\left(R+H^{O}(\Omega), \psi, R\right) & =2 R\left(\frac{1}{\ell}-\frac{1}{\ell *}\right)+\ln \left|\frac{1-\left(1+H^{O}(\Omega) / R\right)\left[1-\frac{1}{2}\left(\frac{\ell}{R}\right)^{2}\right]+\ell * / R}{\left(1+H^{O}(\Omega) / R\right)\left\{\frac{1}{2}\left(\frac{\ell}{R}\right)^{2}+\ell / R\right\}}\right| \\
& \approx 2 R\left(\frac{1}{\ell}-\frac{1}{\ell *}\right)+\ln \left|\frac{\ell *-H^{O}(\Omega)}{\ell}\right| .
\end{aligned}
$$

This is the final simplified form, valid for a small radius $\psi_{0}$ of integration, which can now be studied.

It should be noted that the first term in Eq. (A9) is the leading term, while the second is a corrective term. The leading term converges very rapidly since it holds most of its power in the nearest vicinity of the computation point. For instance, the cumulative sum of this term across a profile gains $\sim 99 \%$ power at $\psi=0.1^{\circ}$. The magnitude of the corrective (logarithmic) term comprises $<1 \%$ of the magnitude of the leading term. One may see that the first terms on the right-hand side of Eqs. (A3) and (A7) are exactly the same. Thus, the difference between the exact expression and the first approximation stems only from the much smaller logarithmic term. As such, our subsequent numerical investigations study the relationship between the term $\ln \left|\frac{1-\left(1+H^{o}(\Omega) / R\right) \cos \psi+\ell^{*} / R}{\left(1+\frac{H^{O}(\Omega)}{R+H^{O}(\Omega)}\right)(1-\cos \psi+\ell / R)}\right|$ and its approximation $\ln \left|\frac{\ell^{*}-H^{o}(\Omega)}{\ell}\right|$.

The variables in both terms are the orthometric height $H^{O}(\Omega)$ and the angular distance $\psi$. The behaviour of these terms within the interval $0.0001^{\circ}(10 \mathrm{~m})<\psi \leq 3^{\circ}$ will 
be numerically investigated. In these tests, the orthometric height takes the following constant values $H^{O}(\Omega)=200 \mathrm{~m}, H^{O}(\Omega)=1 \mathrm{~km}, H^{O}(\Omega)=3 \mathrm{~km}, H^{O}(\Omega)=5 \mathrm{~km}$. In other words, the topography of the test area is assumed to be a plateau with a constant height $H^{O}(\Omega)$.

The logarithmic term is always negative, since the argument $\left|\frac{\ell *-H^{O}(\Omega)}{\ell}\right|$ takes values between 0 and 1, i.e. $0<\left|\frac{\ell *-H^{o}(\Omega)}{\ell}\right|<1$. Figure (A1) shows the behaviour of the logarithmic term and the discrepancies between the exact expression (Eq. A3) and its approximation (Eq. A7) across the integration area. The lower batch of curves in Fig (A1) indicates the magnitude of the logarithmic term for each case, whereas the upper batch denotes the corresponding discrepancies. The test cases in Fig. (A1) are denoted as follows: (a) $H^{O}(\Omega)=200 \mathrm{~m}$ dotted line; (b) $H^{O}(\Omega)=1000 \mathrm{~m}$ dashed line; (c) $H^{O}(\Omega)=2000 \mathrm{~m}$ solid thin line; (d) $H^{O}(\Omega)=5000 \mathrm{~m}$ solid bold line. Also note the negative logarithmic scale.

\section{Figure Al near here}

From Fig. (A1), most of the power in the logarithmic term is also in the nearest vicinity of the computation point and it decreases with increasing $\psi$. Note that at the computation point the discrepancies are almost zero. The magnitude of the relative discrepancies increases linearly with the distance (recall that a logarithmic scale is used in Fig. A1). Note however, that in any tested case and for $\psi<1^{\circ}$ the discrepancies are at least of two orders of magnitude less than the logarithmic term itself. At $\psi=3^{\circ}$, the error of the approximation $\ln \left|\frac{\ell^{*}-H^{o}(\Omega)}{\ell}\right|$ consists of $\sim 3 \%$ only from the exact expression. Also recall that the logarithmic term is $<1 \%$ of the whole Poisson kernel. From the above results, it is obvious that the expression: 
$\bar{K}\left[R+H^{o}(\Omega), \psi\left(\Omega, \Omega^{\prime}\right), R\right]=2 R\left(\frac{1}{\ell}-\frac{1}{\ell^{*}}\right)+\ln \frac{\ell^{*}-H^{o}(\Omega)}{\ell}$ is sufficient as the first approximation of the complicated integration term.

Let us next have a look at the first term in Eq. (A3). Realizing that: $\lim _{\psi \rightarrow 0} \ell *=\sqrt{\ell^{2}+H^{O}(\Omega)^{2}}$, it can be written as:

$2 R\left(\frac{1}{\ell}-\frac{1}{\ell *}\right) \approx 2 R\left(\frac{1}{\ell}-\frac{1}{\sqrt{\ell^{2}+H^{O}(\Omega)^{2}}}\right)=\frac{2 R}{\ell}\left[1-\left(1+\frac{H^{O}(\Omega)^{2}}{\ell^{2}}\right)^{-\frac{1}{2}}\right]$

For $H^{o}(\Omega)<\ell$, (the case of $H^{o}(\Omega)>\ell$ can be treated in a similar way) this can be represented by a convergent binomial series:

$$
2 R\left(\frac{1}{\ell}-\frac{1}{\ell^{*}}\right)=2 R\left[\frac{1}{\ell}-\frac{1}{\ell}\left(1+\frac{H^{o}(\Omega)^{2}}{\ell^{2}}\right)^{-\frac{1}{2}}\right]=2 R\left\{\frac{1}{\ell}-\frac{1}{\ell}\left[1+\sum_{k=1}^{\infty}\left(\begin{array}{c}
-\frac{1}{2} \\
k
\end{array}\right)\left(\frac{H^{o}(\Omega)}{\ell}\right)^{2 k}\right]\right\} .
$$

Carrying out the required algebraic operations, we arrive at:

$2 R\left(\frac{1}{\ell}-\frac{1}{\ell^{*}}\right)=\frac{2 R}{\ell} \sum_{k=1}^{\infty}\left(\begin{array}{c}-\frac{1}{2} \\ k\end{array}\right)\left(\frac{H^{o}(\Omega)}{\ell}\right)^{2 k}$.

The series in Eq. (A10) is alternating, and thus a convergent series even for $H$ and $\ell$ going simultaneously to zero, thus:

$$
\lim _{\substack{\ell \rightarrow 0 \\
H^{O}(\Omega) \rightarrow 0}} 2 R\left(\frac{1}{\ell}-\frac{1}{\ell^{*}}\right)=2 R \lim _{\substack{\ell \rightarrow 0 \\
H^{O}(\Omega) \rightarrow 0}} \frac{1}{\ell} \sum_{k=1}^{\infty}\left(\begin{array}{c}
-\frac{1}{2} \\
k
\end{array}\right)\left(\frac{H^{O}(\Omega)}{\ell}\right)^{2 k}=2 R \lim _{\substack{\ell \rightarrow 0 \\
H^{O}(\Omega) \rightarrow 0}} \frac{1}{\ell} \sum_{k=1}^{\infty}\left(\begin{array}{c}
-\frac{1}{2} \\
k
\end{array}\right) \text { (A14) }
$$

As the summation is a real number, the whole expression grows above all limits and we get:

$\lim _{H \rightarrow 0} 2 R\left(\frac{1}{\ell}-\frac{1}{\ell^{*}}\right)=\delta\left(\Omega, \Omega^{\prime}\right)$,

where $\delta$ is the Kronecker symbol for the function that grows beyond all limits when $\Omega=\Omega$ ' and equals to 0 for all other values of $\Omega$. We can thus see that: 


$$
\begin{aligned}
\lim _{H^{O}(\Omega) \rightarrow 0} \overline{\delta g}{ }^{N T}(\Omega) & =\lim _{H^{O}(\Omega) \rightarrow 0} \frac{1}{4 \pi} \frac{R}{H^{O}(\Omega)} \int_{\Omega^{\prime} \in \Omega_{O}} \bar{K}\left[R+H^{O}(\Omega), \psi\left(\Omega, \Omega^{\prime}\right), R\right] \delta g^{N T}\left(R, \Omega^{\prime}\right) d \Omega^{\prime}= \\
& =\delta g^{N T}(R, \Omega)
\end{aligned}
$$

as one would expect.

We note that the averaging Poisson kernel also has a singularity for the case when $H$ is not equal to zero. For $H^{o}(\Omega)>0$, we get the following equation:

$$
\begin{aligned}
\lim _{\ell \rightarrow 0} 2 R\left(\frac{1}{\ell}-\frac{1}{\ell^{*}}\right) & =\lim _{\ell \rightarrow 0} 2 R\left[\frac{1}{\ell}-\frac{1}{H}\left(1+\frac{\ell^{2}}{H^{o}(\Omega)^{2}}\right)^{-\frac{1}{2}}\right]=\lim _{\ell \rightarrow 0} 2 R\left\{\frac{1}{\ell}-\frac{1}{H^{o}(\Omega)}\left[1+\sum_{k=1}^{\infty}\left(-\frac{1}{2}\right)\left(\frac{\ell}{H^{o}(\Omega)}\right)^{2 k}\right]\right\}= \\
& =\lim _{\ell \rightarrow 0} 2 R\left(\frac{1}{\ell}-\frac{1}{H^{o}(\Omega)}\right)=\lim _{\ell \rightarrow 0} \frac{1}{\ell}
\end{aligned}
$$

which also grows above all limits. Thus, the averaging Poisson kernel has a removable singularity of a linear type (1/0) at the point of interest $\Omega$, whether the height is equal to 0 or not. The second, logarithmic term is always negative: it equals 0 for $\ell$ going to 0 , and it also goes to 0 for growing $\ell$. Note that the argument $0<\left|\frac{\ell *-H^{o}(\Omega)}{\ell}\right|<1$. 
Table 1. Gravity-related notation used throughout this paper.

\begin{tabular}{|c|c|}
\hline$g(r, \Omega)$ & Gravity at an arbitrary point \\
\hline$g^{N T}(r, \Omega)$ & $\begin{array}{l}\text { Gravity generated by masses contained within the geoid, i.e., with the } \\
\text { topography removed and in the NT-space }\end{array}$ \\
\hline$g^{T}(r, \Omega)$ & $\begin{array}{l}\text { Gravitation generated by masses contained within the topography only, } \\
\text { i.e., those between the geoid and Earth's surface }\end{array}$ \\
\hline${ }_{g} \delta \rho(r, \Omega)$ & $\begin{array}{l}\text { Effect on gravitation due to lateral mass-density variations inside the } \\
\text { topography with respect to the reference value of } \rho_{0}=2670 \mathrm{kgm}^{-3}\end{array}$ \\
\hline$\gamma(r, \Omega)$ & Normal gravity generated by the geocentric reference ellipsoid \\
\hline$\delta g^{N T}(r, \Omega)$ & Gravity disturbances generated by masses contained within the geoid \\
\hline$g_{B}^{T}(r, \Omega)$ & Gravitation generated by a spherical Bouguer shell \\
\hline$g_{R}^{T}(r, \Omega)$ & $\begin{array}{l}\text { Gravitation generated by the terrain roughness, i.e., topographical } \\
\text { undulations relative to the spherical Bouguer shell }\end{array}$ \\
\hline $\mathcal{E}_{g}^{-}(\Omega)$ & $\begin{array}{l}\text { Correction to Helmert's approximation of integral-mean gravity along } \\
\text { the plumbline }\end{array}$ \\
\hline$\varepsilon_{H^{o}}(\Omega)$ & $\begin{array}{l}\text { Correction to Helmert's orthometric height to convert it to the rigorous } \\
\text { orthometric height (Tenzer et al. 2005) }\end{array}$ \\
\hline
\end{tabular}




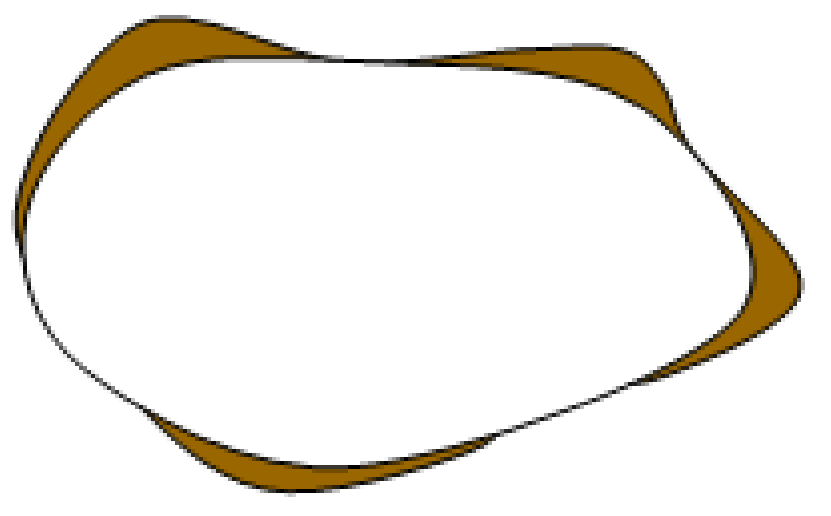

Figure 1. The conceptual decomposition model of actual gravity into the geoidgenerated component (internal white area) and the topography-generated component (dark area); the topography is exaggerated for the sake of clarity. 


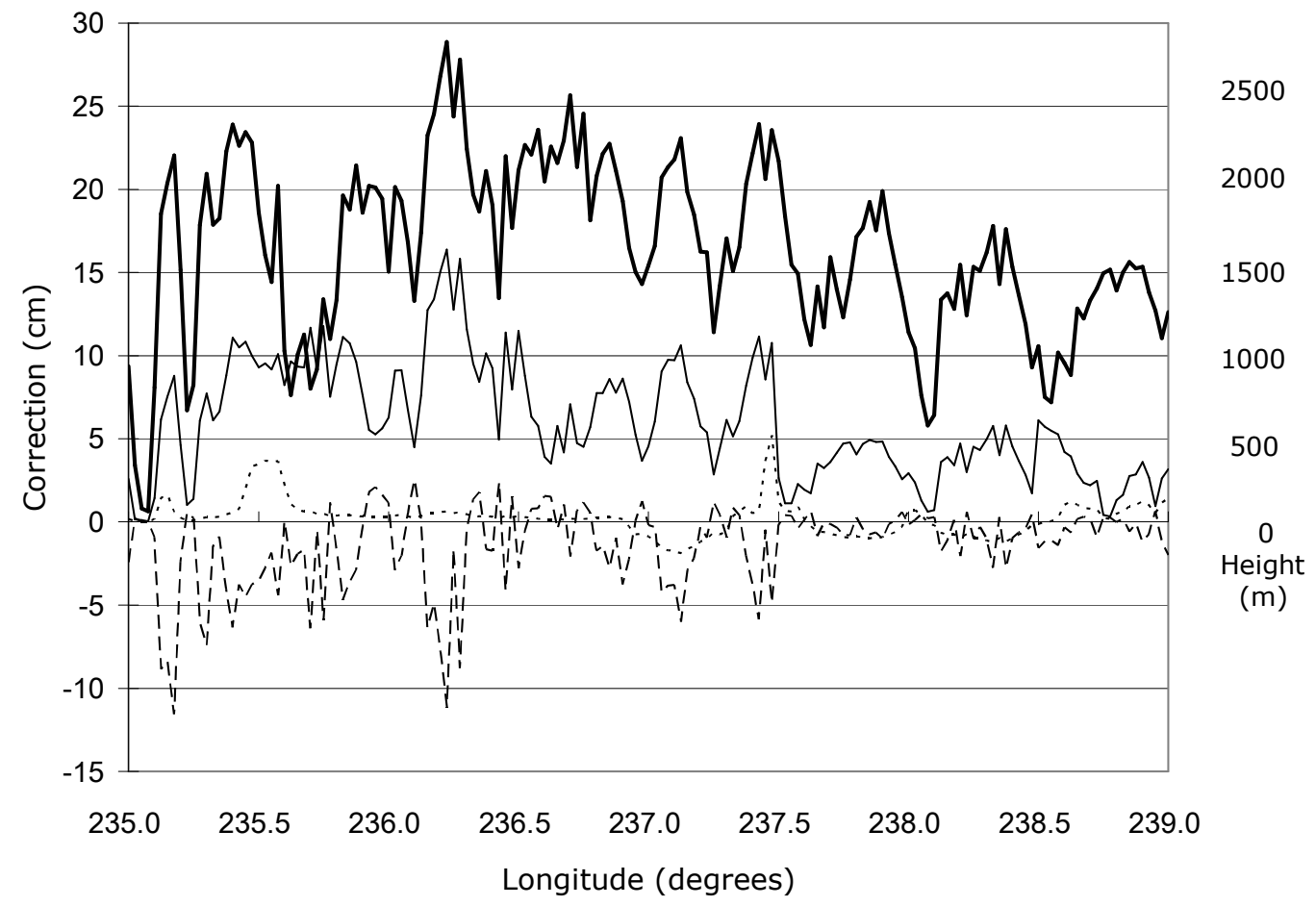

Figure 2. Profiles of the five components of the correction to Helmert's orthometric height $(\mathrm{cm})$, as well as the Helmert orthometric height $(\mathrm{m})$ along a profile at $50^{\circ} \mathrm{N}$. Continuous thick line represents the topographic height; continuous thin line corresponds to $\varepsilon_{H}^{\delta g}{ }_{H}(\Omega)$ [geoidgenerated gravity disturbance]; dashed line corresponds to $\varepsilon_{H}^{T} O(\Omega)$ [terrain-roughnessgenerated gravity]; dotted line corresponds to $\varepsilon_{H}^{\delta \rho}(\Omega)$ [lateral variation of topographical massdensity]. 
Table 2. Descriptive statistics of corrections to Helmert's orthometric height from the profile shown in Fig. 2. Values in $\mathrm{cm}$, rounded to the nearest $\mathrm{mm}$.

\begin{tabular}{|c|c|c|c|c|c|}
\cline { 2 - 6 } \multicolumn{1}{c|}{} & $\begin{array}{c}\text { correction } \\
\text { due to } \\
\text { gravity } \\
\text { disturbance }\end{array}$ & $\begin{array}{c}\text { correction } \\
\text { due to } \\
\text { terrain- } \\
\text { roughness }\end{array}$ & $\begin{array}{c}\text { correction } \\
\text { due to lateral } \\
\text { variation of } \\
\text { density }\end{array}$ & $\begin{array}{c}\text { correction } \\
\text { for 2nd- } \\
\text { order normal } \\
\text { gravity }\end{array}$ & $\begin{array}{c}\text { correction } \\
\text { for 2nd- } \\
\text { order } \\
\text { Bouguer } \\
\text { shell }\end{array}$ \\
\hline Mean & 6.0 & -1.4 & 0.2 & -0.0 & -0.0 \\
\hline STD & 3.3 & 2.5 & 1.1 & 0.0 & 0.0 \\
\hline Minimum & 0.0 & -11.5 & -1.9 & 0.0 & 0.0 \\
\hline Maximum & 15.4 & 2.5 & 5.2 & -0.1 & -0.0 \\
\hline
\end{tabular}




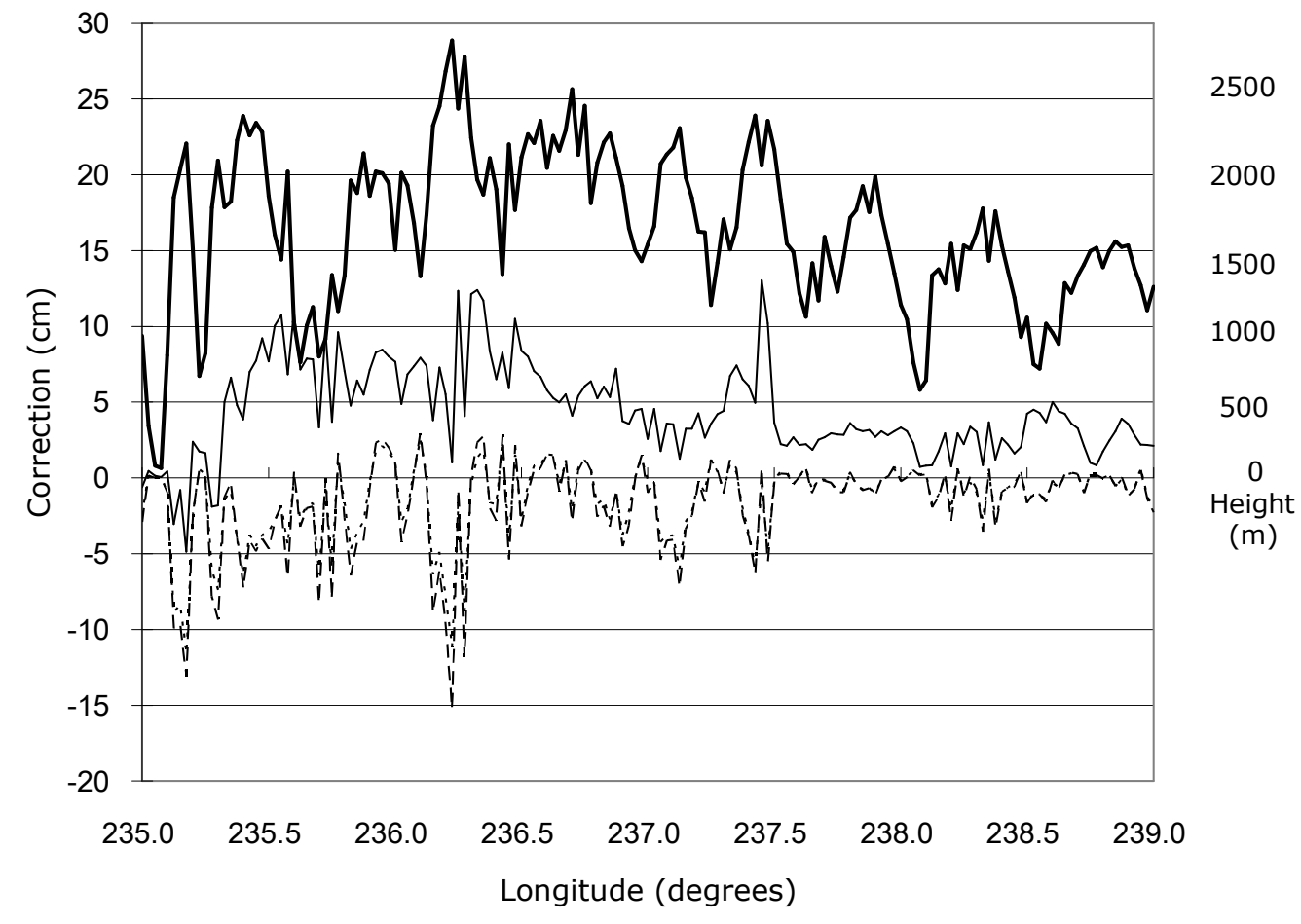

Figure 3. Comparison among the rigorous, Mader and Niethammer corrections $(\mathrm{cm})$ to Helmert orthometric heights along the same profile as in Fig. 2. Continuous thicker line represents the topographic profile; continuous thin line represents the rigorous correction; dashed line represents Niethammer correction; dotted line represents Mader correction. 
Table 3. Descriptive statistics of the total corrections to Helmert's orthometric height from the profile shown in Fig. 3. Values in $\mathrm{cm}$, rounded to the nearest $\mathrm{mm}$.

\begin{tabular}{|c|c|c|c|}
\cline { 2 - 4 } \multicolumn{1}{c|}{} & Mader & Niethammer & Rigorous \\
\hline Mean & -1.4 & -1.7 & 4.4 \\
\hline Standard Deviation & 2.5 & 3.1 & 3.1 \\
\hline Minimum Value & -11.5 & -15.2 & -4.9 \\
\hline Maximum Value & 2.5 & 2.9 & 13.0 \\
\hline
\end{tabular}




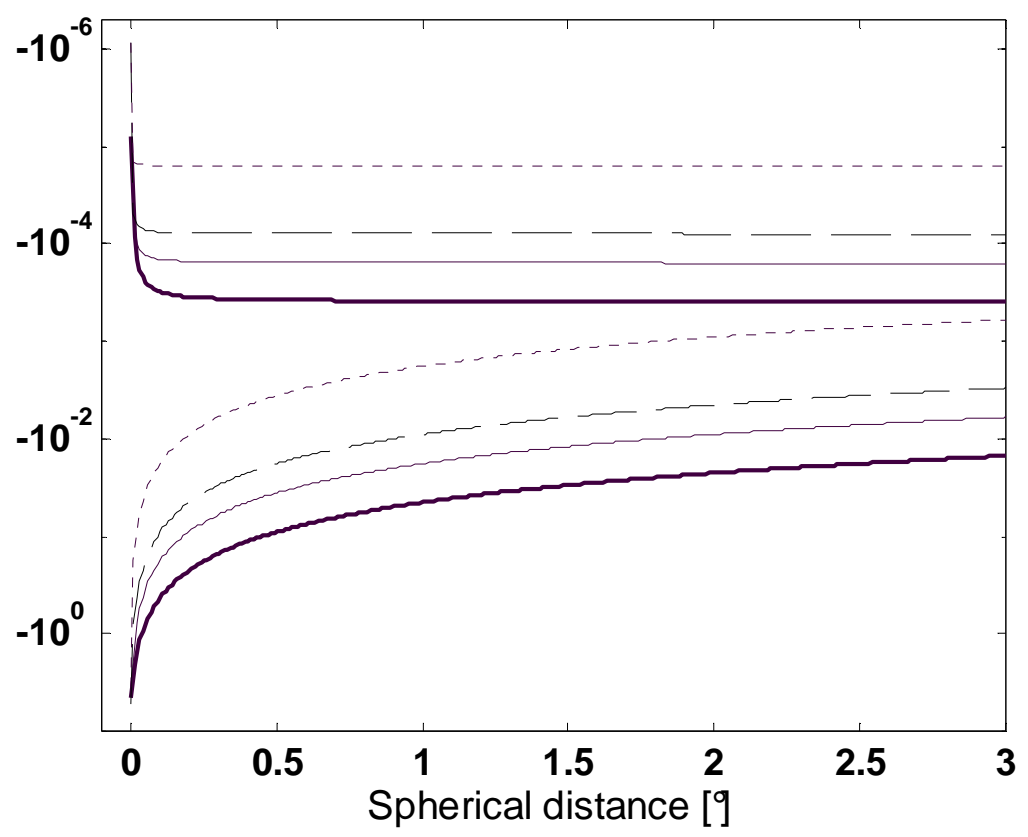

Fig. 1. The behaviour of the $\ln \left|\frac{1-\left(1+H^{o}(\Omega) / R\right) \cos \psi+\ell^{*} / R}{\left(1+\frac{H^{o}(\Omega)}{R+H^{o}(\Omega)}\right)(1-\cos \psi+\ell / R)}\right|$ term (lower batch of curves) and the discrepancies $\ln \left|\frac{1-\left(1+H^{o}(\Omega) / R\right) \cos \psi+\ell^{*} / R}{\left(1+\frac{H^{o}(\Omega)}{R+H^{o}(\Omega)}\right)(1-\cos \psi+\ell / R)}\right|-\ln \left|\frac{\ell^{*}-H^{O}(\Omega)}{\ell}\right|$ (upper batch of curves) 